\title{
AÇÃO PROMOTORA DO ZINCO EM CATALISADORES DE SÍNTESE DO ESTIRENO
}

Hugo Ernane Bonfim Leite, Alcineia Conceição Oliveira e Maria do Carmo Rangel*

Instituto de Química, Universidade Federal da Bahia, Campus Universitário de Ondina, 40170-290 Salvador - BA

Recebido em 12/5/03; aceito em 2/9/03

\begin{abstract}
THE PROMOTER ACTION OF ZINC ON CATALYTS FOR STYRENE SYNTHESIS. Ethylbenzene dehydrogenation in the presence of steam is the main commercial route to produce styrene. The industrial catalyst is chromium and potassium-doped hematite, which easily deactivates with time due to potassium loss. In order to find non-toxic and potassium free catalysts, the promoter action of zinc on hematite was studied in this work. It was found that zinc acts as structural promoter by stabilizing the $\mathrm{Fe}^{3+}$ species (active phase) as maghemite. Although it decreases the specific surface area, it increases four times the catalytic activity as compared to hematite.
\end{abstract}

Keywords: hematite; styrene; ethylbenzene dehydrogenation.

\section{INTRODUÇÃO}

O desenvolvimento de pesquisas relacionadas à produção do estireno vem despertando o interesse da comunidade científica e tecnológica, desde a implantação do primeiro processo comercial em 1930. Isto se deve ao elevado valor agregado deste intermediário químico, amplamente empregado na fabricação de resinas, borrachas sintéticas e polímeros ${ }^{1,2}$.

A principal via comercial de obtenção do estireno é a desidrogenação catalítica do etilbenzeno em presença de vapor d'água, que envolve a reação principal ${ }^{3}$ :

$\mathrm{C}_{6} \mathrm{H}_{5}-\mathrm{CH}_{2} \mathrm{CH}_{3(\mathrm{~g})} \rightleftarrows \mathrm{C}_{6} \mathrm{H}_{5} \mathrm{CH}=\mathrm{CH}_{2(\mathrm{~g})}+\mathrm{H}_{2(\mathrm{~g})} \quad \Delta \mathrm{H}=121 \mathrm{~kJ} \cdot \mathrm{mol}^{-1}$

e as reações secundárias ${ }^{2}$ :

$\mathrm{C}_{6} \mathrm{H}_{5}-\mathrm{CH}_{2} \mathrm{CH}_{3(\mathrm{~g})} \rightleftarrows \mathrm{C}_{6} \mathrm{H}_{6(\mathrm{~g})}+\mathrm{C}_{2} \mathrm{H}_{4(\mathrm{~g})}$

$\mathrm{C}_{6} \mathrm{H}_{5}-\mathrm{CH}_{2} \mathrm{CH}_{3(\mathrm{~g})} \rightleftarrows \mathrm{C}_{6} \mathrm{H}_{5}-\mathrm{CH}_{3(\mathrm{~g})}+\mathrm{CH}_{4(\mathrm{~g})}$

Hidrocarboneto ou coque $\longrightarrow \mathrm{CO}_{2}+\mathrm{y} \mathrm{H}_{2}$

A reação principal é endotérmica e limitada pelo equilíbrio com conversões tipicamente baixas, raramente excedendo $50 \%$, mesmo em processos conduzidos a temperaturas elevadas (em torno de $\left.600{ }^{\circ} \mathrm{C}\right)^{2-4}$.

O vapor d'água possui diversas funções, entre as quais evitar a formação de coque que prejudica o rendimento da reação e danifica o catalisador. $\mathrm{O}$ vapor reage continuamente com os hidrocarbonetos e o coque, que são formados em reações secundárias. Essa remoção resulta num depósito estacionário de carbono $(3 \text { a } 6 \%)^{2}$. Aliado a outros fatores favoráveis, isso permite a operação ininterrupta das unidades comerciais por um a dois anos com a mesma carga. $\mathrm{O}$ vapor também desloca o equilíbrio para a produção de estireno, por meio de redução da pressão parcial do etilbenzeno, além de atuar como agente oxidante, mantendo o catalisador em um estado altamente seletivo ao estireno ${ }^{2,5}$.

Devido à endotermicidade da reação, a temperatura do leito catalítico diminui de modo significativo durante o progresso da rea-

*e-mail: mcarmov@ufba.br ção, em reatores adiabáticos comerciais, resultando em um baixo valor de conversão. Esse decréscimo na temperatura afeta também a seletividade para o estireno, pois, nas condições de equilíbrio, apenas reações indesejáveis ocorrem. Dessa forma, é necessário manter a temperatura em um valor conveniente, de modo a promover alto nível de conversão e alta velocidade de reação. No processo convencional, o aquecimento da corrente do produto, através da adição de vapor superaquecido entre os leitos catalíticos, mantém a temperatura constante ${ }^{2,3,5}$.

Muitos catalisadores já foram testados na desidrogenação do etilbenzeno com vapor d'água. Óxidos de ferro, de alumínio, de cobalto, de estanho, de antimônio, diversos óxidos mistos, zeólitas e materiais mesoporosos e catalisadores suportados mostraram-se ativos e seletivos ao estireno ${ }^{2-15}$. Os óxidos de ferro são os mais empregados comercialmente, devido às suas propriedades catalíticas e ao seu baixo custo. Dessa forma, diversos estudos têm sido direcionados para a melhoria das propriedades texturais e estruturais desses materiais $\mathrm{s}^{2,3,5,7,9,13,16-18}$.

Os catalisadores industriais, de última geração, são sólidos à base de óxido de ferro dopados com óxidos de cromo, de cério e de potássio. Esses sistemas são cataliticamente ativos e seletivos, mas possuem baixas áreas superficiais específicas e vida útil limitada devido à perda de potássio, que migra para o centro da pastilha ("pellet") ou para a saída do reator e é arrastado pela corrente gasosa². Além disso, a toxicidade dos compostos de cromo dificulta o manuseio e o descarte desses materiais. Isto demanda o desenvolvimento de catalisadores isentos de potássio e com áreas superficiais específicas elevadas e estáveis.

Visando alcançar estas características dos sistemas catalíticos, neste trabalho, foi avaliado o efeito do zinco sobre as propriedades catalíticas do óxido de ferro, isento de potássio, destinado à desidrogenação do etilbenzeno, em presença de vapor d'água.

\section{PARTE EXPERIMENTAL}

Os catalisadores foram preparados a partir da hidrólise simultânea de nitrato de ferro e nitrato de zinco $(\mathrm{Zn} / \mathrm{Fe}($ molar $)=1)$, com solução $25 \%$ de hidróxido de amônio, à temperatura ambiente. Os reagentes foram adicionados, por meio de uma bomba peristáltica, a um béquer contendo água, mantendo-se o sistema sob agitação magnética, à temperatura ambiente. Posteriormente à adição dos reagentes, 
o pH foi ajustado para 11 e o sistema foi mantido sob agitação por 30 min adicionais. Em seguida, a solução coloidal foi centrifugada e o gel obtido foi lavado com água, para a remoção do ânion do material de partida, que pode influenciar a atividade catalítica ${ }^{8,13,15,19}$. O material resultante foi centrifugado, lavado com água e novamente centrifugado, até completar um total de seis lavagens. Após a sétima centrifugação, o gel foi seco a $120{ }^{\circ} \mathrm{C}$ por $12 \mathrm{~h}$ e o sólido foi moído e peneirado em 100 mesh. $\mathrm{O}$ material foi calcinado sob fluxo de nitrogênio $(100 \mathrm{~mL} / \mathrm{min})$ a $600{ }^{\circ} \mathrm{C}$, por $2 \mathrm{~h}$, obtendo-se a amostra ZF1. Foram também preparadas amostras de óxido de ferro (amostra F) e de óxido de zinco (amostra $Z$ ), seguindo o mesmo procedimento experimental. Estes materiais foram usados como referência.

Os catalisadores foram caracterizados por espectrometria de emissão atômica em plasma indutivamente acoplado (ICP/AES), medidas de área superficial específica (método BET), difração de raios X (DRX) e redução termoprogramada (TPR). A atividade catalítica foi avaliada na desidrogenação do etilbenzeno, em presença de vapor d'água.

Na determinação dos teores de zinco e ferro, as amostras foram solubilizadas em $10 \mathrm{~mL}$ de ácido clorídrico concentrado, sob refluxo, por 30 min, e depois aquecidas até quase à secura. Em seguida, foram retomadas em solução de ácido clorídrico $1 \%$, transferidas para balões volumétricos de $100 \mathrm{~mL}$, dos quais foram retiradas alíquotas de $10 \mathrm{~mL}$. Estas foram transferidas para outros balões volumétricos de $100 \mathrm{~mL}$, avolumando-os com água. As soluções resultantes foram analisadas em um espectrômetro de emissão atômica em plasma indutivamente acoplado seqüencial, marca ARL, modelo 3410, com minitocha, potência incidente de $650 \mathrm{~W}$, potência refletida inferior a $5 \mathrm{~W}$ e nebulizador concêntrico Meinhard.

Nas medidas de área superficial específica, utilizou-se uma massa de cerca de $0,15 \mathrm{~g}$ que foi acondicionada em uma cela de vidro e aquecida sob fluxo de nitrogênio $(60 \mathrm{ml} / \mathrm{min})$, até $160{ }^{\circ} \mathrm{C}$, por $1 \mathrm{~h}$. Em seguida, a amostra foi analisada com uma mistura $30 \% \mathrm{~N}_{2} / \mathrm{He}$ (60 ml/min). Os perfis de redução termoprogramada foram obtidos no mesmo equipamento, empregando-se uma massa de $0,35 \mathrm{~g}$ e acompanhando-se o consumo de hidrogênio de uma mistura de $5 \% \mathrm{H}_{2} / \mathrm{N}_{2}$, desde 30 até $1000^{\circ} \mathrm{C}$. As amostras foram previamente tratadas sob fluxo de nitrogênio a $150^{\circ} \mathrm{C}$, por $1 \mathrm{~h}$.

Os difratogramas de raios $\mathrm{X}$ foram obtidos através de um instrumento Shimadzu, modelo XD3A, utilizando-se radiação $\mathrm{CuK}_{\alpha}$, gerada a $30 \mathrm{kV}$ e $20 \mathrm{~mA}$ com filtro de níquel. As amostras foram moídas em um almofariz de ágata e, em seguida, acondicionadas no porta-amostra.

Os catalisadores foram avaliados em teste microcatalítico, operando a $530{ }^{\circ} \mathrm{C}$ e $1 \mathrm{~atm}$, na desidrogenação do etilbenzeno em presença de vapor d'água. O arranjo experimental está mostrado na Figura 1. Utilizou-se uma massa de $0,3 \mathrm{~g}$ da amostra e uma vazão de $0,2 \mathrm{ml} / \mathrm{min}$ de etilbenzeno em nitrogênio. $\mathrm{O}$ reator, contendo o catalisador, foi aquecido sob fluxo de nitrogênio até à temperatura da reação. Em seguida, o fluxo de nitrogênio foi interrompido e o reator foi alimentado com uma mistura de etilbenzeno e vapor d'água, obtida pela passagem de uma corrente de nitrogênio por um saturador contendo etilbenzeno. O produto da reação foi recolhido em um condensador na saída do reator, e a fase orgânica foi analisada em um cromatógrafo CG-35, com coluna carbowax 5\% suportada em cromosorb e detetor de ionização de chama. Após os testes catalíticos, analisou-se o teor de $\mathrm{Fe}^{+2}$ dos catalisadores, dissolvendo-se as amostras em ácido clorídrico, sob refluxo, em atmosfera de monóxido de carbono e titulando-as com dicromato de potássio ${ }^{20}$.

\section{RESULTADOS E DISCUSSÃO}

Os resultados de análise química são mostrados na Tabela 1. Nota-

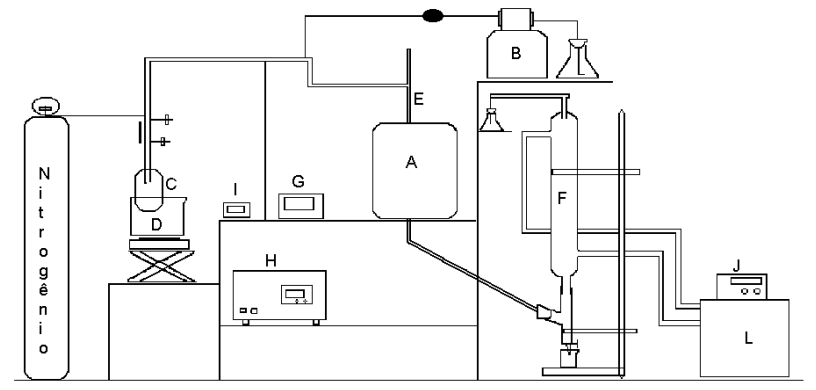

Figura 1. Esquema do teste microcatalítico empregado na avaliação dos catalisadores na desidrogenação do etilbenzeno em presença de vapor d'água. $A=$ forno; $B=$ bomba peristáltica; $C=$ saturador; $D=$ banho de óleo; $E=$ reator; $F=$ condensador; $G=$ indicador da temperatura do forno; $H=$ controlador da temperatura; $I=$ indicador de temperatura do saturador; $J=$ controlador de temperatura do banho de água; $L=$ banho de água; $M=$ cilindro de nitrogênio

se que o método experimental utilizado conduziu a uma maior precipitação dos compostos de ferro, em relação aos de zinco, uma vez que foi obtido um sólido com razão molar $\mathrm{Zn} / \mathrm{Fe}$ inferior àquela da solução de partida.

Tabela 1. Análise química dos catalisadores obtidos. Amostra F: óxido de ferro; amostra Z: óxido de zinco; amostra ZF10: óxido de ferro contendo zinco

\begin{tabular}{lcccc}
\hline Amostra & $\begin{array}{c}\% \mathrm{Fe} \\
( \pm 0,05)\end{array}$ & $\begin{array}{c}\% \mathrm{Zn} \\
( \pm 0,05)\end{array}$ & $\begin{array}{c}\mathrm{Zn} / \mathrm{Fe} \\
(\text { esperado })\end{array}$ & $\begin{array}{c}\mathrm{Zn} / \mathrm{Fe} \\
(\text { obtido })\end{array}$ \\
\hline $\mathrm{F}$ & 71,82 & - & - & - \\
$\mathrm{Z}$ & - & 72,30 & - & - \\
$\mathrm{ZF} 1$ & 47,28 & 47,54 & 1,00 & 0,86 \\
\hline
\end{tabular}

Os difratrogramas de raios $\mathrm{X}$ dos catalisadores à base de óxido de ferro indicaram a presença da hematita $\left(\alpha-\mathrm{Fe}_{2} \mathrm{O}_{3}\right)$, independente da presença do dopante, enquanto a amostra $\mathrm{Z}$ apresentou um perfil típico do óxido de zinco ( $\mathrm{ZnO}$ ). A Figura 2 ilustra os padrões obtidos. Durante o teste catalítico, as amostras de óxido de ferro sofreram transição de fase para formar magnetita $\left(\mathrm{Fe}_{3} \mathrm{O}_{4}\right)$ ou maguiemita $\left(\gamma-\mathrm{Fe}_{2} \mathrm{O}_{3}\right)$, como mostram os difratogramas de raios $\mathrm{X}$ dos catalisadores antes e após a reação (Figuras 2 e 3). As Tabelas 2 e 3 mostram as distâncias interplanares calculadas a partir dos difratogramas. Como se pode observar, não é possível distinguir a presença isolada de magnetita ou maguiemita, já que as distâncias interplanaras calculadas coincidem com os valores das fichas cristalográficas das duas fases. Por outro lado, o óxido de zinco puro não sofreu mudança de fase, durante a desidrogenação do etilbenzeno.

Os valores de área superficial específica dos catalisadores são mostrados na Tabela 4. O material constituído de óxido de ferro puro (amostra F) apresentou a área superficial específica mais elevada (17 $\mathrm{m}^{2} / \mathrm{g}$ ), enquanto o óxido de zinco mostrou o valor mais baixo, entre os catalisadores estudados. A presença de zinco nos catalisadores causou uma diminuição da área superficial específica, indicando que esse metal favorece a sinterização dos sólidos. Isto pode ser explicado admitindo-se que a presença do zinco diminui a rigidez da rede do óxido de ferro, devido ao menor potencial eletrostático das espécies $\mathrm{Zn}^{2+}$, quando comparado ao potencial eletrostático das espécies $\mathrm{Fe}^{3+}$, aumentando a mobilidade das espécies para a sinterização. Após o teste catalítico, todos os catalisadores apresentaram áreas superficiais específicas mais baixas, indicando que a transformação de 


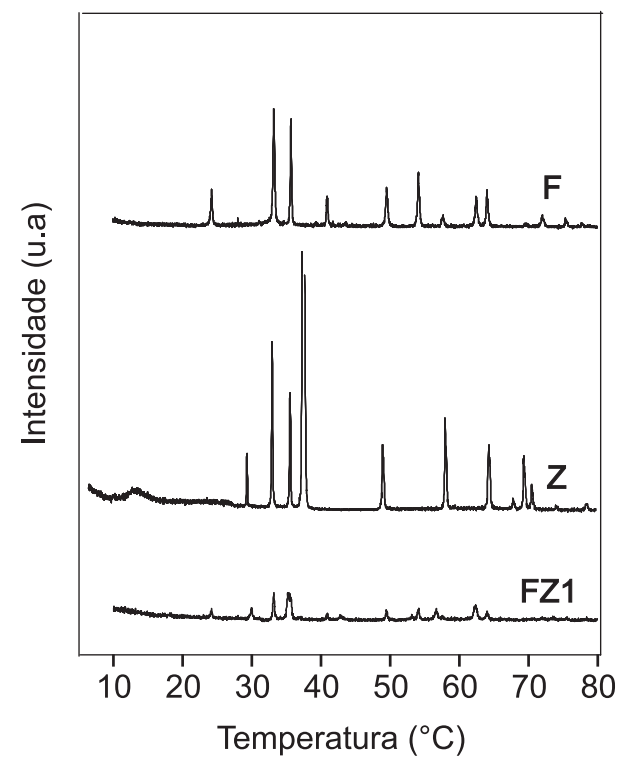

Figura 2. Difratogramas de raios $X$ dos catalisadores novos. Amostra F: óxido de ferro; amostra Z: óxido de zinco; amostra ZF10: óxido de ferro contendo zinco

Tabela 2. Distâncias interplanares $(d)$ dos catalisadores novos, calculadas a partir dos difratogramas de raios X. Amostra F: óxido de ferro; amostra Z: óxido de zinco; amostra ZF1: óxido de ferro contendo zinco

\begin{tabular}{ccccc}
\hline $\begin{array}{c}\text { Ficha } \\
\text { № 86-0550 }\end{array}$ & $\begin{array}{c}\text { Ficha } \\
\text { № 36-1451 } \\
\text { JCPDS 2000 } \\
\text { JCPDS 2000 }\end{array}$ & \multicolumn{3}{c}{ Catalisadores novos } \\
Hematita & Óxido de zinco & F & Z & ZF1 \\
\hline 3,6823 & 2,8143 & 3,71 & 2,79 & 3,72 \\
2,6992 & 2,6033 & 2,67 & 2,56 & 2,71 \\
2,5177 & 2,4759 & 2,56 & 2,49 & 2,57 \\
2,2066 & 1,9111 & 2,20 & 1,89 & 2,25 \\
2,0783 & 1,6247 & 2,12 & 1,61 & - \\
1,8411 & 1,4771 & 1,82 & 1,46 & 1,82 \\
1,6945 & 1,4071 & 1,70 & 1,40 & 1,70 \\
1,6365 & 1,3781 & 1,63 & 1,38 & - \\
1,5987 & 1,3582 & - & 1,36 & 1,59 \\
1,4861 & - & 1,48 & - & 1,47 \\
1,4536 & - & 1,45 & - & 1,4537 \\
1,3111 & - & - & - & 1,3105 \\
1,2274 & - & - & - & 1,2241 \\
\hline
\end{tabular}

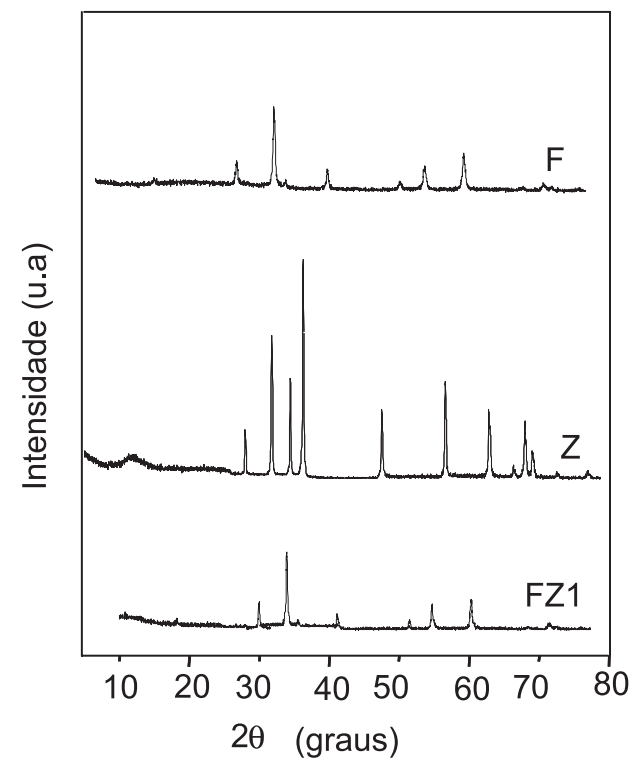

Figura 3. Difratogramas de raios $X$ dos catalisadores usados na reação de desidrogenação do etilbenzeno. Amostra F: óxido de ferro; amostra Z: óxido de zinco; amostra ZF10: óxido de ferro contendo zinco

hematita para magnetita ou maguiemita foi acompanhada da coalescência de partículas e de poros.

Os perfis de redução termoprogramada das amostras são mostrados na Figura 4. A curva de óxido de ferro puro (amostra F) apresentou um pico a $380{ }^{\circ} \mathrm{C}$ devido à redução da hematita para formar a magnetita e outro, a valores mais elevados (cerca de $610{ }^{\circ} \mathrm{C}$ ), atribuído à produção de ferro metálico ${ }^{21}$. A presença de zinco deslocou o pico de formação da magnetita para temperaturas mais altas $\left(450{ }^{\circ} \mathrm{C}\right)$, indicando que este metal dificulta a redução da hematita. Entretanto, a formação do ferro metálico não foi afetada pela presença do zinco. A curva de TPR do óxido de zinco puro não mostrou picos de redução na faixa de temperatura do experimento.

Todos os catalisadores foram ativos na desidrogenação do etilbenzeno, em presença de vapor d'água, como mostram os dados da Tabela 4. A amostra contendo zinco apresentou o melhor desempenho. Isso pode ser atribuído a um aumento da atividade intrínseca, como indicam os valores de atividade por área. Sendo os catalisadores sob estudo mássicos, a atividade intrínseca pode ser convenientemente expressa pela atividade por área. A ação do zinco, em aumentar a atividade intrínseca pode ser atribuída à capacidade desse metal em estabilizar a espécie $\mathrm{Fe}^{+3}$, que forma a fase ativa na reação ${ }^{2,7}$. Isto

Tabela 3. Distâncias interplanares $(d)$ dos catalisadores usados, calculadas a partir dos difratogramas de raios X. Amostra F: óxido de ferro; amostra Z: óxido de zinco; amostra ZF1: óxido de ferro contendo zinco

\begin{tabular}{ccccc}
\hline $\begin{array}{c}\text { Ficha № 88-0315 } \\
\text { JCPDS 2000 }\end{array}$ & $\begin{array}{c}\text { Ficha № 25-1402 } \\
\text { JCPDS 2000 } \\
\text { Magnetita }\end{array}$ & $\begin{array}{c}\text { Ficha № 36-1451 } \\
\text { JCPDS 2000 } \\
\text { Óxido de zinco }\end{array}$ & F & \multicolumn{2}{c}{$\begin{array}{c}\text { Catalisadores usados } \\
\text { Z }\end{array}$} \\
\hline 4,8353 & 4,82 & 2,8143 & 4,92 & 2,88 \\
2,9610 & 2,95 & 2,6033 & 2,98 & 2,63 \\
2,5251 & 2,51 & 2,4759 & 2,56 & 2,43 \\
2,0937 & 2,08 & 1,9111 & 2,10 & 1,89 \\
1,7095 & 1,70 & 1,6247 & 1,73 & 1,61 \\
1,6117 & 1,60 & 1,4771 & 1,63 & 1,49 \\
1,4805 & 1,47 & 1,4071 & 1,47 & 1,73 \\
1,3242 & 1,31 & 1,3781 & $-1,39$ & 1,62 \\
1,2771 & 1,27 & 1,3582 & 1,28 & 1,36 \\
\hline
\end{tabular}


Tabela 4. Área superficial específica dos catalisadores novos $(\mathrm{Sg})$ e usados $\left(\mathrm{Sg}^{*}\right)$.na desidrogenação do etilbenzeno. Amostra F: óxido de ferro; amostra Z: óxido de zinco; amostra ZF1: óxido de ferro e zinco

\begin{tabular}{ccc}
\hline Amostras & $\mathrm{Sg}\left(\mathrm{m}^{2} / \mathrm{g}\right)$ & $\mathrm{Sg}^{*}\left(\mathrm{~m}^{2} / \mathrm{g}\right)$ \\
\hline $\mathrm{F}$ & 17 & 11 \\
$\mathrm{Z}$ & 3,0 & 1,0 \\
$\mathrm{ZF} 1$ & 11 & 6,0 \\
\hline
\end{tabular}

foi confirmado pelos resultados de redução termoprogramada, assim como pelos valores da razão $\mathrm{Fe}^{+2} / \mathrm{Fe}^{+3}$ dos sólidos, medidos após o teste catalítico. Como se pode observar na Tabela 4, após a reação, o óxido de ferro apresenta um valor próximo ao valor estequiométrico da magnetita, enquanto o material contendo zinco mostra um valor inferior, indicando a formação de uma mistura de magnetita e maguiemita. Devido ao valor dessa razão, pode-se supor que a maior parte do óxido de ferro presente está sob a forma da maguiemita, justificando a maior estabilidade das espécies $\mathrm{Fe}^{3+}$. A seletividade do catalisador não foi alterada pela presença do zinco, indicando que os materiais, com e sem dopante, possuem sítios ativos de mesma natureza.

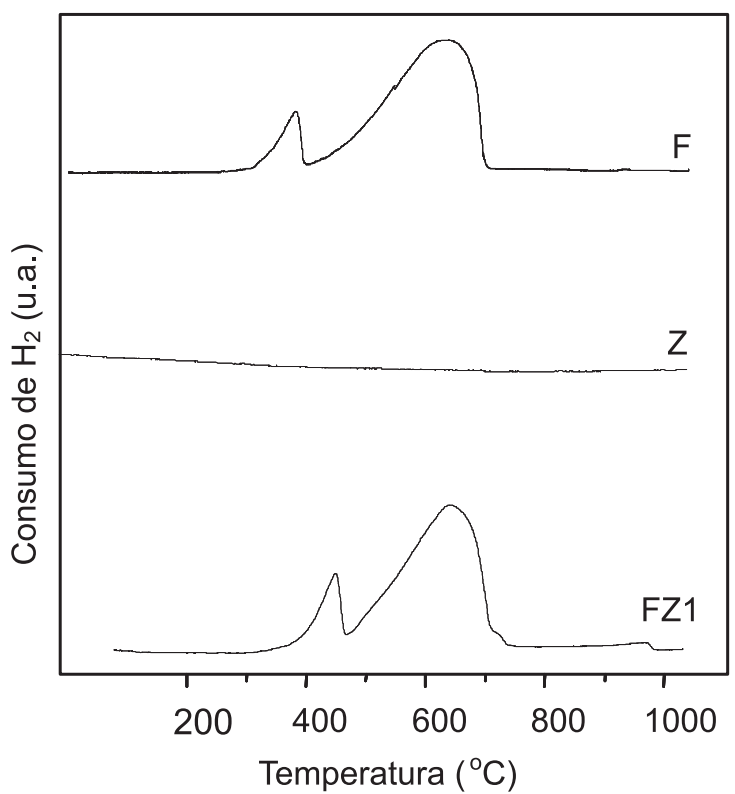

Figura 4. Perfil de redução termoprogramada os catalisadores. Amostra F: óxido de ferro; Amostra Z: óxido de zinco; Amostra ZF10: óxido de ferro contendo zinco

Tabela 5. Conversão (C), atividade (a), atividade por área $(\mathrm{a} / \mathrm{Sg})$, seletividade $(\mathrm{S})$ dos catalisadores na desidrogenação do etilbenzeno e razão $\mathrm{Fe}^{+2} / \mathrm{Fe}^{+3}$ dos catalisadores após a reação. Amostra F: óxido de ferro; amostra Z: óxido de zinco; amostra ZF1: óxido de ferro e zinco

\begin{tabular}{cccccc}
\hline Amostras & $\mathrm{C}(\%)$ & $\begin{array}{c}\mathrm{a} \mathrm{x} \mathrm{10} \\
\left(\mathrm{mol} \mathrm{s}^{-1} \mathrm{~g}^{-1}\right)\end{array}$ & $\begin{array}{c}\mathrm{a} / \mathrm{Sg} \times 10^{8} \\
\left(\mathrm{~mol} \mathrm{~s}^{-1} \mathrm{~g}^{-1}\right)\end{array}$ & $\mathrm{S}(\%)$ & $\mathrm{Fe}^{+2} / \mathrm{Fe}^{+3}$ \\
\hline $\mathrm{F}$ & 3,50 & 7,0 & 6,0 & 92,0 & 0,5 \\
$\mathrm{Z}$ & 0,60 & 1,0 & 10 & 41,0 & - \\
\hline
\end{tabular}

\section{CONCLUSÂO}

O zinco atua como promotor estrutural em catalisadores de óxido de ferro, destinados à desidrogenação do etilbenzeno, em presença de vapor d'água, para produzir estireno. Essa ação promotora foi atribuída à capacidade do zinco para estabilizar a espécie $\mathrm{Fe}^{+3}$ (fase ativa do catalisador), através da formação da maguiemita $\left(\gamma-\mathrm{Fe}_{2} \mathrm{O}_{3}\right)$. A amostra contendo zinco mostrou-se promissora para a reação, apresentando uma atividade quatro vezes maior $\left(\mathrm{a}=28 \times 10^{-7} \mathrm{~mol} \mathrm{~s}^{-1} \mathrm{~g}^{-1}\right)$ que a de um catalisador de óxido de ferro puro $\left(\mathrm{a}=7 \times 10^{-7} \mathrm{~mol} \mathrm{~s}^{-1} \mathrm{~g}^{-1}\right)$. As seletividades dos dois catalisadores foram próximas.

\section{AGRADECIMENTOS}

H. E. B Leite agradece ao PIBIC/CNPq pela bolsa de iniciação científica e A. C. Oliveira agradece ao CNPq pela bolsa de mestrado concedida. Os autores agradecem o apoio financeiro da FINEP e $\mathrm{CNPq}$.

\section{REFERÊNCIAS}

1. Kirk, R. E.; Othmer, R. F.; Encyclopedia of Chemical Techonology, ed. Wiley Interscience: New York, 1984, vol. 15, p. 775.

2. Lee, E. H. ; Catal. Rev. Sci. Eng. 1973, 8, 285.
3. Hirano, T.; Appl. Catal. 1988, 40, 247

4. Li, W. Y.; Lettman, C.; Maier, W. F.; Catal. Lett. 2000, 69, 181.

5. Styles, A. B. Em Applied Industrial Catalysis; Leach, B. E., ed.; Academic Press: New York, 1987, p. 137.

6. Bispo, J. R.; Oliveira, A. C.; Correa, M. L.; Marchetti; S. G.; Fierro, J. L. G.; Rangel, M. C.; Stud. Surf. Sci. Catal. 2002, 142, 517.

7. Meima, G. R.; Menon, P. G.; Appl.Catal. 2001, 212, 239.

8. Wong, S.; Lin, H.; Mou, C.; Appl. Catal. 2000, 198, 103.

9. Miyakoshi, A.; Ueno, A.; Ichikawa, M.; Appl.Catal. 2001, 219, 249.

10. Vislovskiy, V. P.; Chang, J.; Park, M.; Park, S.; Catal. Commun. 2002, 3, 227.

11. Miyakoshi, A.; Ueno, A.; Ichikawa, J.; Appl.Catal. 2001, 216, 137.

12. Chen, Q.; Chen, X.; Mao, L.; Cheng, W.; Catal. Today 1999, 51, 141.

13. Oliveira, A. C.; Fierro, J. L.G.; Rangel, M. C.; Catal. Today 2003, 85, 49.

14. Oliveira, A. C., Rangel, M. C.; Quim. Nova 2003, 26, 170.

15. Oliveira, A. C.; Valentini, A.; Nobre, P. S. S.; Rangel, M. C.; React. Kinet. Catal. Lett. 2002, 75, 1.

16. Araújo, G. C.; Souza, A. O.; Pinheiro, E. A.; Rangel, M. C.; Quim. Nova 2002, 25, 181

17. Quadro, E. B.; Dias, M. L; Amorim, A. M. M.; Rangel, M. C.; J. Braz. Chem. Soc. 1999, 10, 51.

18. Quadro, E. B.; Souza, M. O.; Rangel, M. C.; Quim. Nova 1998, 21, 428.

19. Sreekumar, K.; Mathew, T.; Bevassy, M. B.; Raygopal, R.; Netrivel, R.; Raso, B. S.; Appl. Catal. 2001, 205, 11.

20. Vogel, A. I.; Quantitative Inorganic Analysis, ed. Longman: London, 1961, p. 309.

21. González, J. C.; González, M. C.; Laborde, M. A.; Moreno, N.; Appl. Catal. 1986, 20, 3 